\title{
Immune checkpoint inhibitors in MITF family translocation renal cell carcinomas and genetic correlates of exceptional responders
}

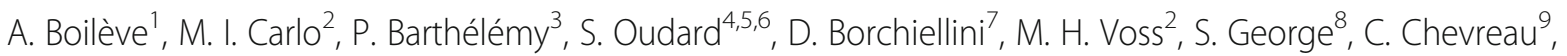 \\ J. Landman-Parker ${ }^{10}$, M-D Tabone ${ }^{10}$, D. D. Chism ${ }^{11}$, A. Amin ${ }^{12}$, M. A. Bilen ${ }^{13}$, D. Bosse ${ }^{14}$, A. Coulomb-L'hermine ${ }^{15}$, \\ Xiaoping Su${ }^{16}$, T. K. Choueiri ${ }^{14}$, Nizar M. Tannir ${ }^{17^{*}}$ and Gabriel G. Malouf ${ }^{1,3,18,19^{*}}$
}

\begin{abstract}
Background: Microphthalmia Transcription Factor (MITF)family translocation renal cell carcinoma (tRCC) is a rare RCC subtype harboring TFE3/TFEB translocations. The prognosis in the metastatic $(\mathrm{m})$ setting is poor. Programmed death ligand-1 expression was reported in $90 \%$ of cases, prompting us to analyze the benefit of immune checkpoint inhibitors $(\mathrm{ICl})$ in this population.

Patients and methods: This multicenter retrospective study identified patients with MITF family mtRCC who had received an $\mathrm{ICl}$ in any of 12 referral centers in France or the USA. Response rate according to RECIST criteria, progression-free survival (PFS), and overall survival (OS) were analyzed. Genomic alterations associated with response were determined for 8 patients.
\end{abstract}

Results: Overall, 24 patients with metastatic disease who received an $\mathrm{ICl}$ as second or later line of treatment were identified. Nineteen (82.6\%) of these patients had received a VEGFR inhibitor as first-line treatment, with a median PFS of 3 months (range, 1-22 months). The median PFS for patients during first ICI treatment was 2.5 months (range, 1-40 months); 4 patients experienced partial response $(16,7 \%)$ and $3(12,5 \%)$ had stable disease. Of the patients whose genomic alterations were analyzed, two patients with mutations in bromodomain-containing genes (PBRM1 and BRD8) had a clinical benefit. Resistant clones in a patient with exceptional response to ipilimumab showed loss of BRD8 mutations and increased mutational load driven by parallel evolution affecting 17 genes (median mutations per gene, 3), which were enriched mainly for O-glycan processing $\left(29.4 \%, F D R=9.7 \times 10^{-6}\right)$.

Conclusions: MITF family tRCC is an aggressive disease with similar responses to ICls as clear-cell RCC. Mutations in bromodomain-containing genes might be associated with clinical benefit. The unexpected observation about parallel evolution of genes involved in O-glycosylation as a mechanism of resistance to $\mathrm{ICl}$ warrants exploration.

Keywords: TFE3, TFEB, Antiangiogenic agents, O-glycosylation, Bromodomain-containing genes, Parallel evolution

\footnotetext{
* Correspondence: ntannir@mdanderson.org; gabriel.malouf@chru-

strasbourg.fr

${ }^{17}$ Department of Genitourinary Medical Oncology, The University of Texas

MD Anderson Cancer Center, Houston, TX, USA

'Department of Medical Oncology, Hôpital Universitaire Pitié-Salpétrière,

Paris, France

Full list of author information is available at the end of the article
}

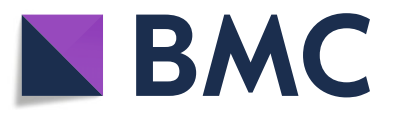

(c) The Author(s). 2018 Open Access This article is distributed under the terms of the Creative Commons Attribution 4.0 International License (http://creativecommons.org/licenses/by/4.0/), which permits unrestricted use, distribution, and

reproduction in any medium, provided you give appropriate credit to the original author(s) and the source, provide a link to the Creative Commons license, and indicate if changes were made. The Creative Commons Public Domain Dedication waiver (http://creativecommons.org/publicdomain/zero/1.0/) applies to the data made available in this article, unless otherwise stated. 


\section{Introduction}

Microphthalmia Transcription Factor (MiTF) family translocation renal cell carcinoma (tRCC) is a subtype of RCC characterized by chromosomal translocations involving TFE3 and TFEB transcription factor genes [1]. As tRCCs with TFE3 or TFEB mutations share clinical, histopathological and molecular features, the 2013 ISUP Vancouver classification grouped these entities as the "MiTF/TFE translocation carcinomas family" [2]. The frequency of adult TFE3 tRCC has been reported to range between 1 and 5\% of all RCCs [3-5]. tRCC usually occurs in children, adolescents and young adults, with a high female predominance [3-5]. There are no approved therapies for metastatic tRCC, and effective therapy for this cancer remains an unmet medical need.

The current first-line standard of care for good risk metastatic clear-cell RCC (ccRCC) is the tyrosine kinase inhibitors (TKIs) targeting vascular endothelial growth factor receptor (VEGFR) [6]. Conversely, the combination of ipilimumab and nivolumab is the standard of care for intermediate and poor risk disease [7]. While there is no standard of care for non-clear cell metastatic RCCs (referred to here as non-ccRCC), retrospective analyses indicate that VEGFR-targeted agents provide some efficacy in metastatic tRCC, with an objective response rate of $30 \%$ and a median progression-free survival (PFS) duration of 7.1-8.2 months [8, 9].

Recently, virtual karyotyping of tRCC identified a subgroup with $17 \mathrm{q}$ gain characterized by activation of the cytotoxic $\mathrm{T}$ lymphocyte-associated protein 4 (CTLA4) pathway [10]. Another study exploring programmed death ligand 1 (PD-L1) expression in a wide range of non-ccRCC identified PD-L1 overexpression in tumor-infiltrating immune cells in $90 \%$ of tRCC cases [11]. Those studies prompted us to explore the efficacy of immune checkpoint inhibitors (ICIs) in this setting. Nivolumab, a programmed death 1 (PD-1) checkpoint inhibitor, was associated with longer overall survival (OS) than mTOR inhibitors in a phase III study involving previously treated patients with metastatic ccRCC and is now often used as second-line therapy [12]. Currently, data regarding the efficacy of ICIs in nonccRCC are limited, and results of clinical trials are pending.

The purpose of this study is to determine the efficacy of ICIs in the treatment of tRCC and to correlate tumor genomic alterations with objective response. We performed a retrospective multicenter analysis of the outcomes of patients with tRCC treated with an ICI in 12 institutions in France and the USA. The efficacy of first-line TKI treatment was also analyzed.

\section{Patients and methods}

\section{Patients}

Patients with tRCC were identified through searches of the patient databases of 12 institutions in France and the
USA for the period from July 2011 to May 2017. Inclusion criteria included tRCC diagnosed by immunohistochemical analysis (IHC) and treatment with at least one ICI. A dedicated genitourinary pathologist at each of the participating institutions verified tRCC diagnoses. TFE3 expression was confirmed by IHC analysis in all cases. FISH confirmation was not a requirement in this study, but was available in the majority of cases. Cases that were tested but not confirmed by FISH were excluded. Clinical characteristics and treatment-related outcome data for ICIs (targeting PD-1, PD-L1 or CTLA4), administered alone or in combination with other agents, were retrospectively determined by individual chart review. We collected data concerning prior treatments, first metastasis, date of first treatment, toxic effects, date of progression and date of death or last follow-up contact. All patients' data were anonymized and de-identified prior to analysis. Patient data were collected in compliance with the IRB guidelines of each participating institution. Written informed consent was obtained from all patients for whom genomic testing was performed. All study protocols were performed in accordance with the ethical tenets of the Declaration of Helsinki.

\section{Assessment of tumor response}

Patients were monitored by their physician until the end of treatment. All treatments and responses, from diagnosis to death or loss to follow-up, were recorded. Tumor response and disease progression by RECIST 1.1 criteria were documented. Stable disease was defined as a stable RECIST response for more than 3 months. Clinical benefit was defined as Miao et al. and included patients with partial response or stable disease lasting more than 6 months [13].

\section{Genomic analysis}

Targeted sequencing data on 410 cancer genes using MSK-IMPACT were collected on tumors from 4 cases, with a median coverage of 580x per case (range, 2301141) [14]. Whole-exome sequencing was performed on another 4 tumors and matched normal adjacent tissues. Briefly, exomes were captured using Agilent SureSelect Human All Exon $50 \mathrm{Mb}$ (Agilent Technologies, Santa Clara, CA, USA) according to the manufacturer's instructions. The technical details and mutation detection method were as previously described [15]. Median coverage obtained for tumor samples was $\sim 100 x$. Mutational load was defined as the total number of somatic mutations obtained per whole-exome sequencing. To compare the mutational load of these tRCCs with mutational load in ccRCC, somatic mutations of ccRCC cases from The Cancer Genome Atlas (TCGA) were retrieved from a report on ccRCC published by TCGA [16]. 


\section{Statistical analysis}

Study endpoints were response rates according to RECIST criteria PFS, and OS. The Kaplan-Meier method was used for survival analyses. PFS was measured from the date of initiation of ICI treatment to the time of progression at any site or death from any cause. All statistical analyses were done by using GraphPad Prism (GraphPad Software, La Jolla, CA, USA).

\section{Results}

\section{Patient characteristics}

Overall, we identified 24 patients who met the inclusion criteria. Selected demographic and clinical characteristics of these patients are summarized in Tables 1 and 2. Before receiving an ICI, the majority of patients had received a VEGFR-targeted agent as first-line therapy (Fig. 1).

\section{Clinical outcomes: First-line VEGFR-targeted agents}

Median PFS for first-line TKI therapy was 3 months (range, 1-22 months) (Fig. 2a). Partial responses were observed in 2 patients (10.5\%), and 15 patients exhibited disease progression at the time of the first interim assessment. Six patients received an mTOR inhibitor (2, first line; 4, second line or later) and none achieved objective response. The toxic effects of sunitinib, the most frequently received first-line agent $(n=15)$, were comparable overall to those reported in studies in RCC and included mainly asthenia and rash.

\section{Clinical outcomes: First immune checkpoint inhibitor}

Of the 24 patients, 17 received nivolumab, 3 received ipilimumab and 4 received ICI-based combination therapy (Table 2). All patients received at least one dose of an ICI; 22 (91.6\%) received 4 doses or more. The median PFS was 2.5 months (range, 1-40 months) (Fig. 2b). Four patients $(16,6 \%)$ experienced a partial response and 3 $(12,5 \%)$ had stable disease in response to the ICI. Among the four patients who achieved an objective response, one received pembrolizumab in combination with a 41BB agonist [17] (PFS 30 months), two received nivolumab (PFS 8 and 3 months) and one received ipilimumab (PFS 9 months). Remarkably, one of the 5 responders, patient 1, showed partial response to ipilimumab lasting for 9 months. At the time of ipilimumab administration, this patient had an ECOG performance status (PS) of 3, with peritoneal, liver and lung metastases. His ECOG PS improved quickly on ipilimumab therapy, leading to a complete response of his abdomen and lung metastases; a residual $6 \mathrm{~cm}$ mediastinal mass was resected. The patient achieved partial response 4 months after starting ipilimumab, but developed bilateral grade 4 optic neuropathy, as previously described [14]. Upon progression, he began treatment with nivolumab, but 6 weeks later his disease had progressed, including development of 8
Table 1 Selected baseline demographic and clinical characteristics of 24 patients with metastatic MITF family translocation renal cell carcinoma treated with an immune checkpoint inhibitor

\begin{tabular}{lll}
\hline Characteristics & Number of patients & Percentage of patients \\
\hline Sex & 4 & 20 \\
Male & 20 & 80 \\
Female & & \\
Age, years & 34 & \\
Median & $3-79$ & \\
Range & 11 & 46 \\
$\leq 34$ & 13 & 54 \\
$>34$ & & \\
Karnofsky score & 9 & 40 \\
$\leq 80$ & 13 & 60 \\
$>80$ & & 88 \\
Translocation type & 21 & 12 \\
TFE3 & 3 & \\
TFEB &
\end{tabular}

Common site of metastasis

Lymph nodes $\quad 15 \quad 63$

Lung $\quad 8 \quad 33$

$\begin{array}{lll}\text { Liver } & 8 & 33\end{array}$

$\begin{array}{lll}\text { Bone } & 8 & 33\end{array}$

Heng score

0 (favorable) $\quad 2 \quad 8$

$1-2$ (intermediate) $18 \quad 75$

3-4- 5 (poor) $\quad 4 \quad 17$

First-line therapy

$\begin{array}{lll}\text { Sunitinib } & 15\end{array}$

$\begin{array}{lll}\text { Pazopanib } & 4 & 17\end{array}$

$\begin{array}{lll}\text { Sorafenib } & 1 & 4\end{array}$

m-TOR inhibitor 2

High dose IL2 $\quad 1 \quad 4$

metastatic lesions in the brain. Genomic evolution of the tumor of this exceptional responder is reported below. The most frequent toxic effects of the ICIs, except for patient 1 , were asthenia grade $2(n=9)$ and dyspnea grade $2(n=3)$. With a median follow-up duration of 19.3 months, the median OS was 24 months. Of note, no pseudoprogression was observed among the 24 patients.

\section{Genomic correlates of response to $\mathrm{ICI}$}

Tumor genomic was available in 8 patients treated with ICIs, four had whole exome sequencing and four targeted sequencing. Four of these patients (50\%) derived clinical benefit from the ICI, including 2 patients with partial response and 2 patients with stable disease. Median interval 


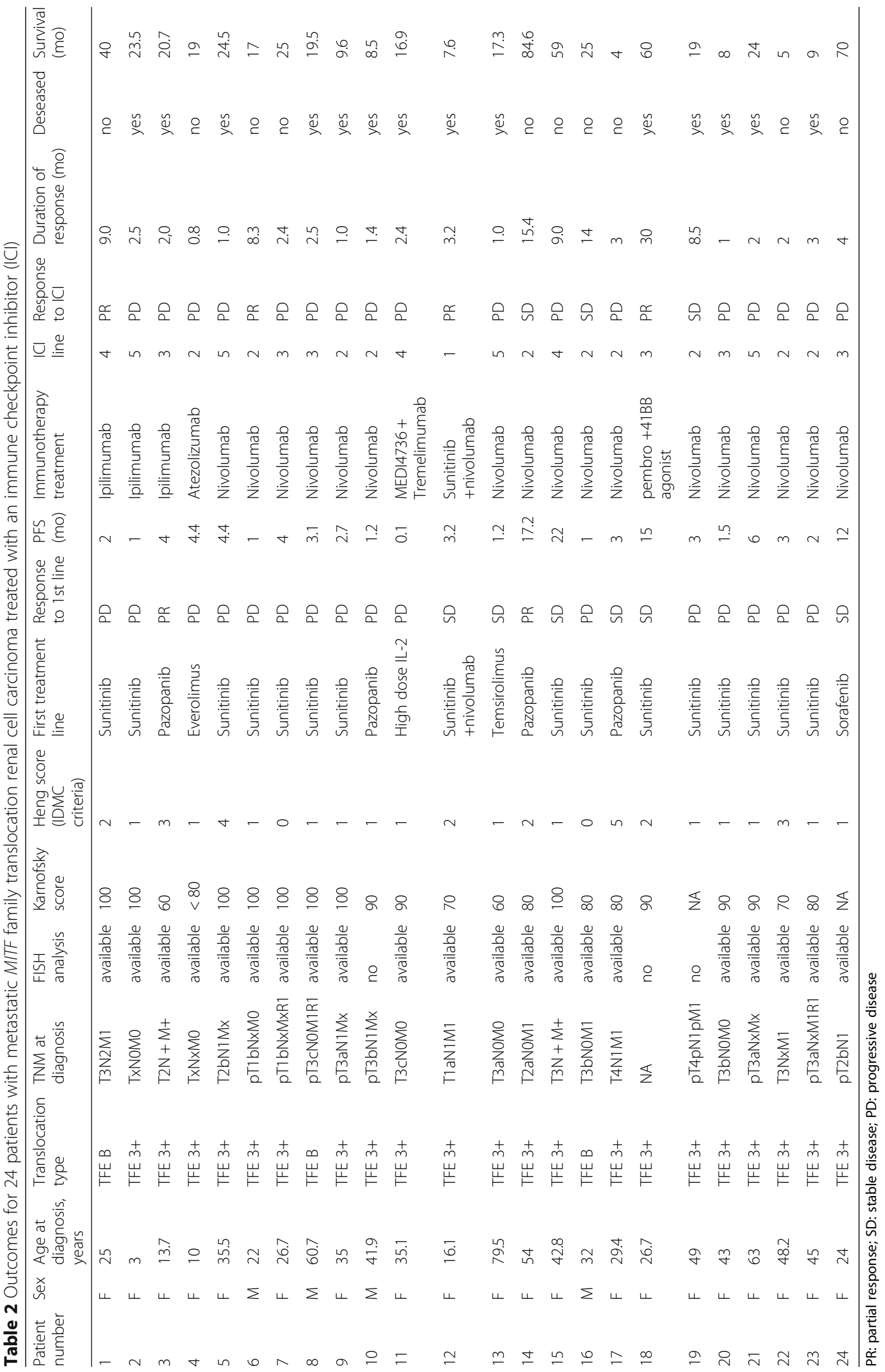




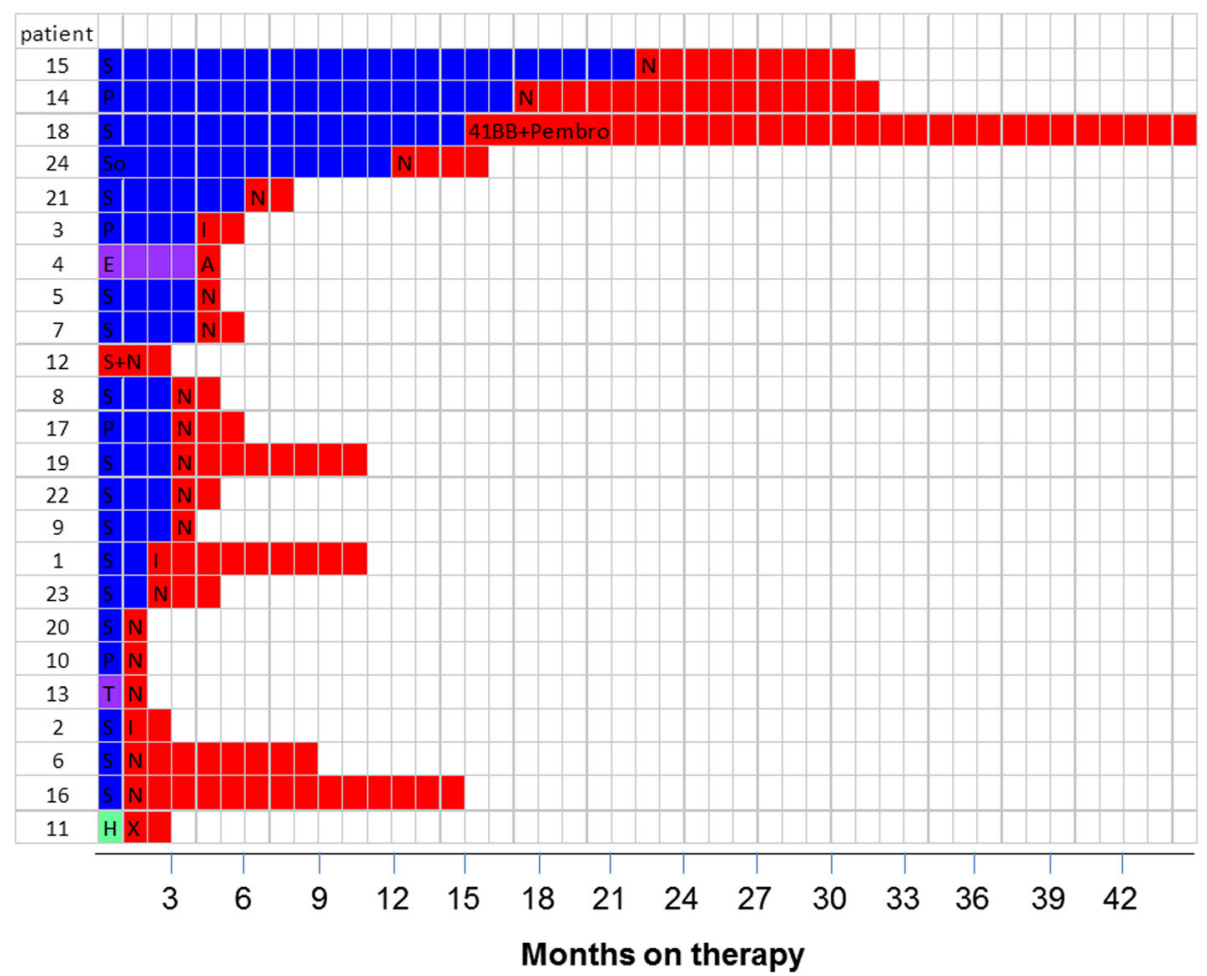

Fig. 1 Responses to tyrosine kinase inhibitors and immune checkpoint inhibitors in 24 patients with metastatic MITF family translocation renal cell carcinoma. Blue/purple/cyan: first-line therapy; red: second-line therapy. S, sunitinib; P, pazopanib; So, sorafenib; N, nivolumab; I, ipilimumab; A, atezolizumab; X, combination MEDI4736 + tremelimumab; 41BB + Pembro, 41 BB agonist and pembrolizumab; E, everolimus; T, temsirolimus; $\mathrm{H}$, high-dose IL2; NA, Not available

time between NGS and start of TKI was 3.8 months (range: 0.4-50 months).

The mutational load of the 4 tumors assessed by whole exome sequencing was low, ranging from 4 to 30 mutations per exome. No recurrent mutation was identified by exome sequencing (Fig. 3a). Overall, the median mutational load of these 4 tRCCs was lower than that of the
ccRCC samples from the TCGA dataset $(n=424 ; p<$ 0.0001) (Fig. 3b). Focusing on the 410 cancer genes covered by both MSK-IMPACT and whole-exome sequencing in all samples, the median mutation rate in the 8 tumors was 0 (range, 0-3). Notably, SMARCA4 mutation was the sole recurrent mutation, identified in 2 cases. The two patients which showed clinical benefit
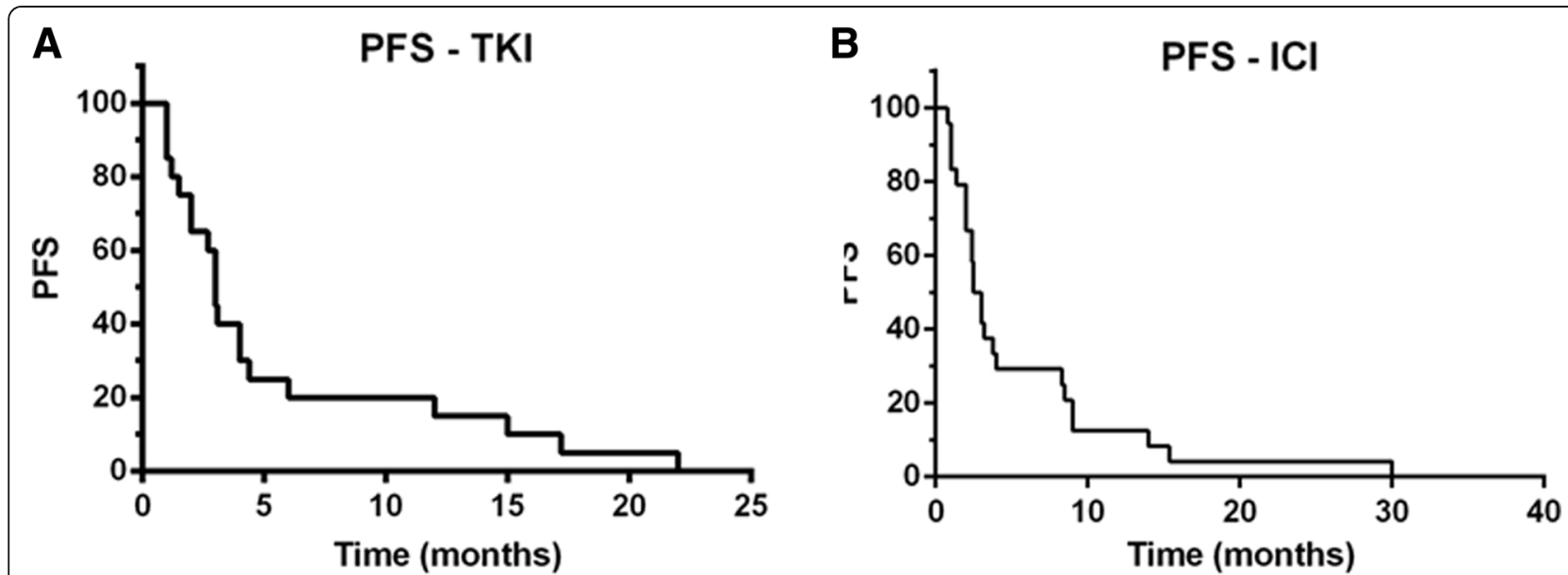

Fig. 2 Kaplan-Meier curves for progression-free survival (PFS) of patients with metastatic MITF family translocation renal cell carcinoma treated with (a) a tyrosine kinase inhibitor (TKI) or (b) an immune checkpoint inhibitor (ICI) 


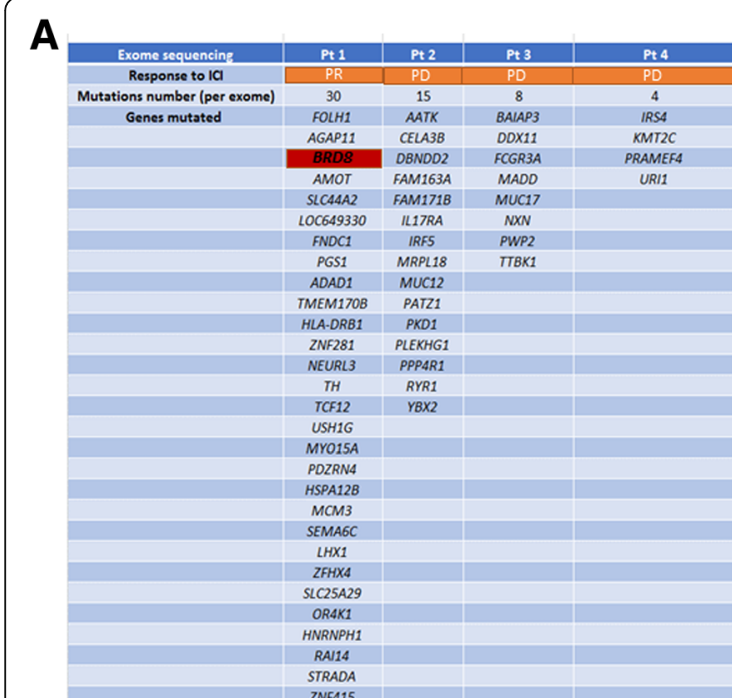

B
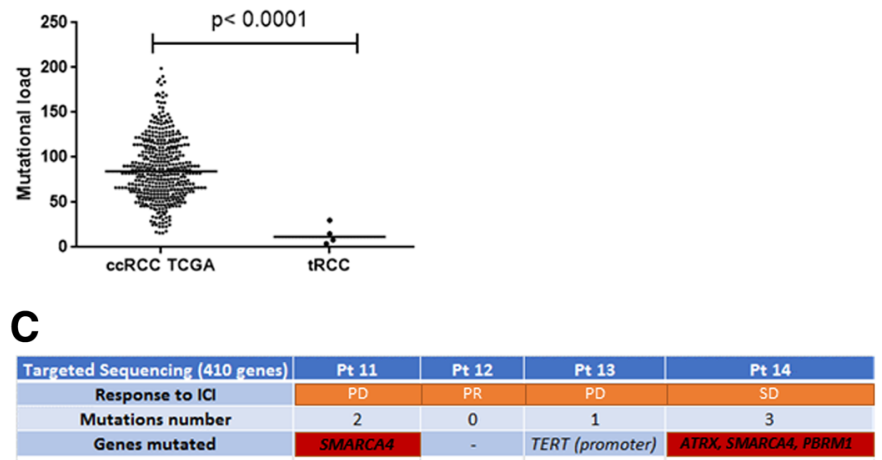

Fig. 3 Genomic correlates of response to immune checkpoint inhibitor (ICI) therapy in a subset of 8 patients with metastatic MITF family translocation renal cell carcinoma (tRCC). The identified mutations and mutational load were assessed by either whole-exome sequencing or targeted sequencing. Numbers of mutations and genes mutated in each sample are given. a Genes mutated in the 4 samples assessed by whole-exome sequencing. $\mathbf{b}$ Box-plots depicting mutational load in tRCC patients $(n=4)$ assessed by whole-exome sequencing as compared to that in patients with clear-cell RCC (ccRCC) from the TCGA dataset $(n=420)$. c Genes mutated in the 4 samples assessed by targeted sequencing

lasting for at least 6 months harbored mutations of bromodomain member genes (PBRM1 and BRD8) (Fig. 3c), consistent with a recently reported association between mutations of bromodomain genes response to ICIs [18].

\section{Genomic landscape of resistant clones in a patient with exceptional response}

As already described, patient 1 developed a dramatic response to ipilimumab lasting for 9 months; the patient had a complete response except for one resistant clone that was stable under treatment with ipilimumab, which was resected 9 months after the last ipilimumab administration and subjected to whole-exome sequencing at 2 distinct opposite regions. The number of somatic mutations in these 2 resistant clones was high, ranging from 120 to 136 mutations $/ 50 \mathrm{Mb}$ as compared to 30 mutations $/ 50 \mathrm{Mb}$ in the primary tumor (Fig. 4a). The majority of mutations present in the primary tumor $(n=25 ; 83.3 \%)$ were also present in both resistant clones, suggesting branched tumor evolution; surprisingly, the BRD8 mutation was lost in both resistant clones. Unexpectedly, we also discovered a phenomenon of parallel evolution of somatic mutations involving 17 distinct genes, with a median of 3 somatic mutations per gene (range, 2-13) (Fig. 4b-c). Gene Ontology analysis using String identified enrichment of O-glycan processing genes $\left(n=5\right.$; false discovery rate $\left.=9.7 \times 10^{-6}\right)$ (Fig. 4b), strongly suggesting the importance of this pathway in the acquired resistance to ICI in this exceptional responder. $C D C 27$ was the most frequently mutated gene, involving 13 and 14 single-nucleotide polymorphisms in resistant clones 1 and 2, respectively (Fig. 4c).

\section{Discussion}

In this international, multicenter retrospective study of 24 patients with metastatic MITF family tRCC who received ICI therapy, we found that $16,7 \%$ of patients had a clinical response to an ICI, with a disease control rate of $29 \%$ when stable disease was also included. Although genetic assessment was available for limited number of samples, we discovered that tumors of patients with clinical benefit harbored mutations in bromodomaincontaining genes. This is, to our knowledge, the first assessment of the clinical efficacy of ICIs in patients with this type of RCC.

The lack of standard treatment for patients with metastatic tRCC is due mainly to the exclusion of patients with non-ccRCC from most large randomized trials; only a few small trials have included tRCC patients, all grouped with non-ccRCCs. Given the benefits of nivolumab in ccRCC, and the lack of other effective therapies for non-ccRCCs, this ICI is being used increasingly in non-ccRCC, although with few data to support its efficacy. Nivolumab is approved in the second-line setting for patients with RCC who have received a VEGFRtargeted agent, based on the results of Checkmate 025, a randomized phase III trial comparing nivolumab to everolimus [12]. Patients treated with nivolumab had a longer OS (25.0 vs 19.6 months) and greater response rate $(25 \%$ vs $5 \%)$, although no difference in PFS was observed. However, no patients with non-ccRCC were included in that study.

Some preliminary data support the use of ICIs in non-ccRCC. Choueiri et al. reported a series of patients 


\section{A}

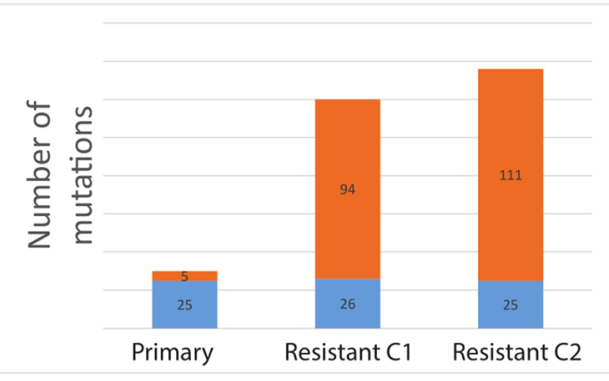

B

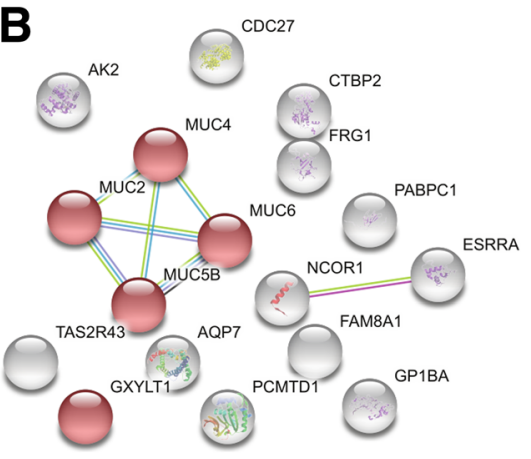

C

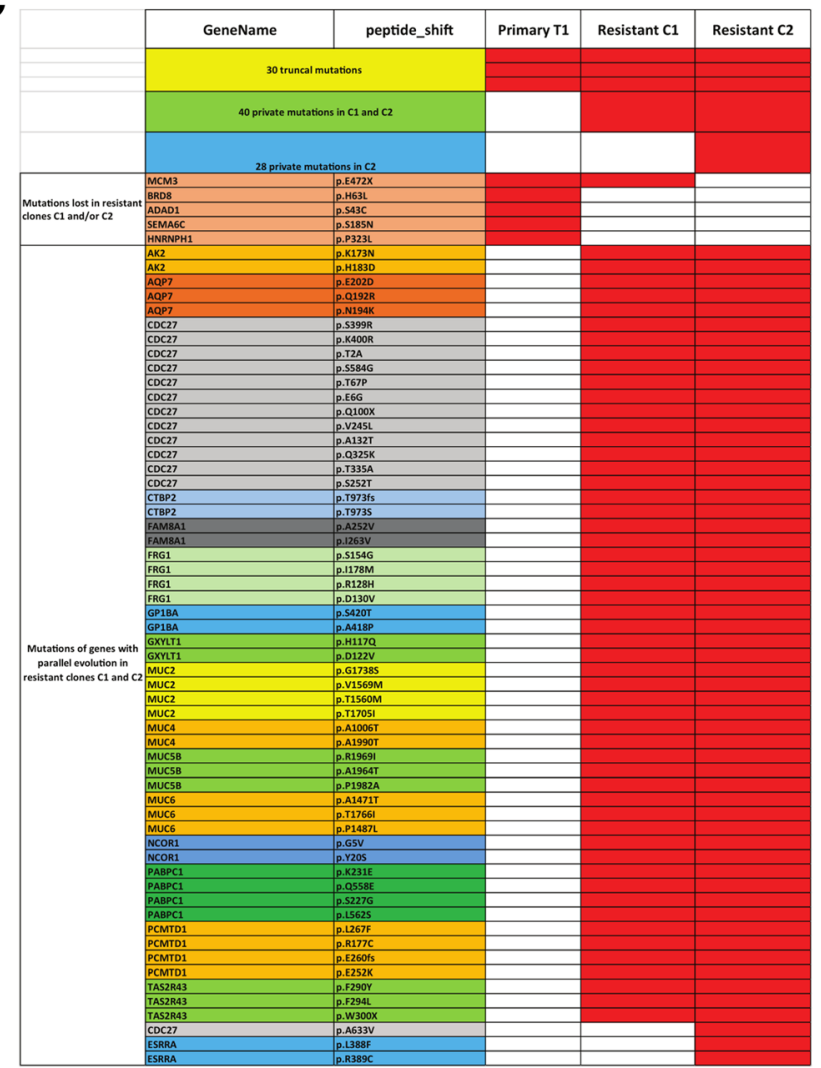

Fig. 4 Genomic evolution of a tumor from a patient who had an exceptional response to ipilimumab. a Numbers of somatic mutations in the primary tumor and in the 2 resistant clones following ipilimumab treatment reveal an increase of mutational load. Blue indicates shared mutations across all 3 samples; orange indicates private mutations. b String network analysis of 17 genes showing parallel evolution reveals 5 genes (in red) linked to the O-glycosylation process. c List of somatic mutations in the primary tumor and resistant clones showing mutations lost in resistant clones as compared to primary tumor and mutations in genes with parallel evolution

with non-ccRCC whose tumors and tumor-infiltrating mononuclear cells were analyzed for PD-L1 by IHC [11]. Of the 10 patients with tRCC, 3 were shown to have PD-L1+ tumor cells and 9 PD-L1+ tumor-infiltrating cells. Two small retrospective series have reported on a combined 81 patients with non-ccRCC treated with an ICI $[19,20]$. Although only 4 patients with tRCC were included in those studies, one patient had a partial response, one had stable disease, and 2 had progressive disease.
Our study considerably expands what is known about the outcomes of ICI therapy for metastatic tRCC patients. As expected, most of the patients we identified (71\%) were treated with nivolumab. These patients' median PFS, 3 months, was shorter than the 4.6 months reported for CheckMate 025, although it is generally understood that PFS is not an optimal measure to gauge benefit from nivolumab therapy [12]. Similarly, overall response rate was $16,7 \%$, compared to $25 \%$ in CheckMate 025. To date, no predictive biomarkers have been 
approved for selecting RCC patients who will best respond to ICIs, although several markers have been explored [21]. Higher tumor mutational load has been correlated with response to ICIs in several tumor types $[22,23]$. Our data showing a low mutational load in tRCC confirmed previous reports; the limited mutational load in tRCC, even in metastatic cases, suggests low numbers of neoantigens in these tumors. The retrospective nature and small sample size of this analysis precludes any conclusions of the predictive value for any genomic event. It is, however, important to highlight here that the two patients lasting clinical benefit harbored somatic mutations of bromodomain-containing genes PBRM1 and BRD8. Recently, mutations of PBRM1 have been shown to be associated with benefit from nivolumab in patients with ccRCC [13]. Interestingly, one of the responder received pembrolizumab in combination with a $41 \mathrm{BB}$ agonist, a costimulatory molecule induced upon TCR activation that promotes cell survival and enhances cytotoxic T-cell responses. This combination may have enhanced the efficacy of pembrolizumab.

Notably, this is the first published report, to our knowledge, not only of a loss of BRD8 mutation in the 2 resistant clones in response to an ICI but also of an increase in mutational load and a phenomenon of parallel evolution affecting genes involved in O-glycosylation. Parallel evolution is a mechanism that has been demonstrated in bacteria and plants and is thought to contribute to the selection of key forces that help predict and prepare for the organism's future evolutionary course [24]. Given the major role of glycosylation in adaptive immune activation [25], further studies are needed to clarify the importance of this process in ICI response. Furthermore, unbiased genomic screens showed recently that dysfunction of $C D C 27$, a member of the anaphasepromoting complex/cyclosome, limits excessive instability of cancer chromosomes, allowing tumor cells to dynamically improve their fitness during cancer evolution [26]. Notably, the high rate of somatic mutations found in the $C D C 27$ gene suggests that this might provide a selective advantage, improving fitness and limiting genetic instability. Reporting genomic results of exceptional responders to immunotherapy have been shown to provide much information to explore mechanisms of immunotherapy sensitivity and resistance. For example, PTEN mutation and reduced expression of genes encoding neoantigens was recently identified as potential mediators of resistance to immune checkpoint therapy in one patient with metastatic uterine leiomyosarcoma who had experienced complete tumor remission for $>2$ years on anti-PD-1 monotherapy [27]. In addition, long term responses to anti-PD1 immunotherapy was recently described in four patients with small cell carcinoma of the ovary, a highly aggressive monogenic cancer driven by
SMARCA4 mutations [28]; this was unexpected for a low mutation burden cancer, but the majority of the tumors demonstrated PD-L1 expression with strong associated T-cell infiltration [28].

The majority of the patients in our series received a VEGFR-targeted agent as first-line therapy prior to the ICI, with disappointing results. Two small retrospective series have specifically looked at response to VEGFR-targeted agents in tRCC $[8,9]$. In one series of patients with metastatic tRCC treated with a VEGFR- or mTORtargeted agent, the median PFS of the 21 patients who received sunitinib was 8.2 months ( $95 \%$ confidence interval, 2.6-14.7) [9]. In another series of 15 patients treated with a variety of VEGFR-targeted agents, the median PFS was 7.1 months, with 3 achieving a partial response [8]. The median PFS durations in these studies were considerably longer than that in our cohort. Although the small numbers of patients limit comparison, the earlier studies, which used TFE3 staining to confirm the diagnosis, may have included patients without a true translocation, whereas in this study the majority of cases (87.5\%) were confirmed by FISH confirmation of translocation. Given that VEGFR-targeted therapies are still used as first-line treatment for RCC, further studies should be conducted to confirm the efficacy of these agents with molecular or FISH correlation of translocation.

Despite being one of the largest retrospective reviews, the small number of patients is the main limitation of our study. The small cohort is partly explained by the rarity of this subtype of RCC. Another limitation is that our cohort included patients with different ages at onset who received different ICIs and combinations. However, it is the first multicenter study of consecutive patients treated in several centers of expertise across Europe and the USA.

\section{Conclusion}

In summary, ICI showed objective response in TRCC similar to those observed in clear-cell RCC. New studies are needed to explore factors associated with resistance in this setting. Mutations in bromodomain-containing genes might predict response to ICIs as reported in other cancer subtypes, and this requires prospective exploration. Importantly, responses to VEGFR-targeted agents also appear to be limited in this subtype, with a shorter PFS than previously reported, and a few durable responses were seen with ipilimumab or combination therapies [18, 20]. Given the early data showing high rates of response to combinations of an ICI and a VEGFR-targeted agent in patients with ccRCC, combinations are now being explored in clinical trials in non-ccRCC, including tRCC [NCT02724878, NCT02496208]. When available and due to rarity of this population, these trials should be considered for patients with MITF family tRCC. Development and studies of novel, biology-driven agents are crucially needed. 


\section{Abbreviations}

ccRCC: Clear-cell renal cell carcinoma; CTLA4: Cytotoxic T lymphocyteassociated protein 4; ICl: Immune checkpoint inhibitors; IHC: Immunohistochemical analysis; m: Metastatic; non-ccRCC: Non clear-cell renal cell carcinoma; OS: Overall survival; PD-1: Programmed death 1; PDL1: Programmed death ligand 1; PFS: Progression-free survival;

PS: Performance status; RCC: Renal cell carcinoma; TCGA: The Cancer Genome Atlas; TKI: Tyrosine kinase inhibitors; tRCC: Translocation renal cell carcinoma; VEGFR: Vascular endothelial growth factor receptor

\section{Acknowledgements}

We would like to acknowledge Kathryn Hale from the Department of Scientific Publications of MD Anderson Cancer center for proofreading.

\section{Funding}

This research did not receive any specific grant from funding agencies in the public, commercial, or not-for-profit sectors.

\section{Availability of data and materials}

The datasets used and/or analysed during the current study are available from the corresponding author on reasonable request.

\section{Authors' contributions}

$A B$ and $G M$ designed the study, collected data, performed analysis and interpretation of the data and drafted the manuscript. MC collected, analyzed, performed interpretation of the data and contributed to drafting the manuscript. PB, SO, DB, MV, SG, CC, JL, MT, DC, MB, AA, DB, AC, XS, TC, NT collected data, contributed to interpretating the data and to drafting the manuscript. All authors read and approved the final manuscript.

\section{Ethics approval and consent to participate}

Patient data were collected in compliance with the IRB guidelines of each participating institution. All study protocols were performed in accordance with the ethical tenets of the Declaration of Helsinki.

\section{Consent for publication}

Written informed consent was obtained from all patients for whom genomic testing was performed. Oral informed consent was obtained from all other patients.

\section{Competing interests}

Dr. Malouf reports consultancy fees from BMS, Pfizer, Ibsen, Novartis, Astellas, and grants from Novartis and Pfizer, outside the submitted work. Dr. Barthélémy reports consultancy fees from BMS, Pfizer, Novartis, outside the submitted work. Dr. Chevreau reports consultancy fees from BMS, Ipsen, Pfizer, Novartis,outside the submitted work. Dr. Borchiellini reports consulting fees from Bristol-Myers Squibb, Ipsen, Novartis, Pfizer, outside the submitted work. Dr. Voss reports consulting fees from Exelixis, Novartis, Eisai, Calithera, Alexion, grants from Genentech, BMS, outside the submitted work. Dr. George reports consultancy fees from Bristol-Myers Squibb, Novartis, Bayer, Pfizer, Exelixis, AstraZeneca, Janssen Oncology, Corvus Pharmaceuticals, Genentech/Roche, and research grants from Pfizer, Acceleron Pharma, Merck, Agensys, Novartis, Bristol-Myers Squibb, Bayer. Dr. Chism reports consultancy fees from Karyopharm Inc., Pfizer, Exelixis outside the submitted work. Dr. Tannir reports consulting fees from BMS, Exelixis, Nektar, Novartis, Pfizer, Argos, Calithera and grants from BMS, Exelixis, Epizyme, Novartis, Miranti, outside the submitted work, outside the submitted work. Dr. Choueiri has reports 1) research grants (Institutional and personal) from AstraZeneca, Bayer, BMS, Cerulean, Eisai, Foundation Medicine Inc., Exelixis, Ipsen, Tracon, Genentech, Roche, Roche Products Limited, GlaxoSmithKline, Merck, Novartis, Peloton, Pfizer, Prometheus Labs, Corvus, Calithera, Analysis Group, Takeda. 2) Honoraria: AstraZeneca, Alexion, Sanofi/Aventis, Bayer, BMS, Cerulean, Eisai, Foundation Medicine Inc., Exelixis, Genentech, Roche, GlaxoSmithKline, Merck, Novartis, Peloton, Pfizer, EMD Serono, Prometheus Labs, Corvus, Ipsen, Up-toDate, NCCN, Analysis Group, NCCN, Michael J. Hennessy (MJH) Associates, Inc (Healthcare Communications Company with several brands such as OnClive and PER), L-path, Kidney Cancer Journal, Clinical Care Options, Platform Q, Navinata Healthcare, Harborside Press, American Society of Medical Oncology, NEJM, Lancet Oncology, Heron Therapeutics. • 3) Consulting or Advisory Role: AstraZeneca, Alexion, Sanofi/Aventis, Bayer, BMS, Cerulean, Eisai, Foundation Medicine Inc., Exelixis, Genentech, Heron Therapeutics, Roche,
GlaxoSmithKline, Merck, Novartis, Peloton, Pfizer, EMD Serono, Prometheus Labs, Corvus, Ipsen, Up-to-Date, NCCN, Analysis Group. • No speaker's bureau - No leadership or employment in for-profit companies (other present or past leadership roles: Director of GU Oncology Division at Dana-Farber and past President of medical Staff at Dana-Farber), member of NCCN Kidney panel and the GU Steering Committee, past chairman of the Kidney cancer Association Medical and Scientific Steering Committee) • No Patents, royalties or other intellectual properties - Travel, accommodations, expenses, in relation to consulting, advisory roles, or honoraria - Medical writing and editorial assistance support may have been funded by Communications companies funded by pharmaceutical companies - The institution (Dana-Farber Cancer Institute) may have received additional independent funding of drug companies or/and royalties potentially involved in research around the subject matter.Dr. Boilève, Dr. Carlo, Dr. Oudard, Dr. Landman-Parker, Dr. Tabone, Dr. Amin, Dr. M. A Bilen, Dr. Bosse, Dr. A. Coulomb-L'hermine and Dr. Su have nothing to disclose.

\section{Publisher's Note}

Springer Nature remains neutral with regard to jurisdictional claims in published maps and institutional affiliations.

\section{Author details}

'Department of Medical Oncology, Hôpital Universitaire Pitié-Salpétrière, Paris, France. ${ }^{2}$ Department of Medicine, Memorial Sloan-Kettering Cancer Center, New York, NY, USA. ${ }^{3}$ Service d'Hématologie et d'Oncologie, Centre Hospitalier Universitaire de Strasbourg, Strasbourg, France. ${ }^{4}$ Oncology Department, European Georges Pompidou Hospital, René Descartes University, Paris, France. ${ }^{5}$ Association pour la Recherche sur les Thérapeutiques Innovantes en Cancérologie, Paris, France. ' U790 PARCC, European Georges Pompidou Hospital, René Descartes University, Paris, France. ${ }^{7}$ Centre Antoine Lacassagne, Nice, France. ${ }^{8}$ Department of Medicine, Roswell Park Cancer Institute, Buffalo, NY, USA. ${ }^{9}$ IUCT-Oncopole, Institut Claudius-Regaud, Toulouse, France. ${ }^{10}$ Service d'Hématologie et d'Oncologie Pédiatrique, Hopital Armand-Trousseau, Paris, France. ${ }^{11}$ Division of Hematology and Oncology, Department of Medicine, Vanderbilt Ingram Cancer Center, Vanderbilt University Medical Center, Nashville, TN, USA. ${ }^{12}$ Carolinas Healthcare System, Levine Cancer Institute, Charlotte, NC, USA. ${ }^{13}$ Department of Hematology and Medical Oncology, Winship Cancer Institute of Emory University, Atlanta, GA, USA. ${ }^{14}$ Department of Medical Oncology, Dana-Farber Cancer Institute and Brigham and Women's Hospital, Harvard Medical School, Boston, MA, USA. ${ }^{15}$ Service d'Anatomopathologie, Hôpital Armand-Trousseau, Paris, France. ${ }^{16}$ Department of Bioinformatics and Computational Biology, The University of Texas MD Anderson Cancer Center, Houston, TX, USA. ${ }^{17}$ Department of Genitourinary Medical Oncology, The University of Texas MD Anderson Cancer Center, Houston, TX, USA. ${ }^{18}$ Department of Functional Genomics and Cancer, Institut de Génétique et de Biologie Moléculaire et Cellulaire, Illkirch, France. ${ }^{19}$ Department of Hematology and Oncology, Centre Hospitalier Universitaire de Strasbourg, 1, Place de l'Hôpital, 67000 Strasbourg, France.

Received: 14 August 2018 Accepted: 13 December 2018

Published online: 27 December 2018

\section{References}

1. Kauffman EC, Ricketts CJ, Rais-Bahrami S, et al. Molecular genetics and cellular features of TFE3 and TFEB fusion kidney cancers. Nat Rev Urol. 2014; 11(8):465.

2. Delahunt B, Srigley JR, Egevad L, et al. International Society of Urological Pathology grading and other prognostic factors for renal neoplasia. Eur Urol. 2014;66(5).

3. Classe M, Malouf GG, Su X, et al. Incidence, clinicopathological features and fusion transcript landscape of translocation renal cell carcinomas. Histopathology. 2017;70(7):1089-97.

4. Komai Y, Fujiwara M, Fujii Y, et al. Adult Xp11 translocation renal cell carcinoma diagnosed by cytogenetics and immunohistochemistry. Clin Cancer Res. 2009;15(4):1170-6.

5. Zhong M, De Angelo P, Osborne L, et al. Translocation renal cell carcinomas in adults: a single institution experience. Am J Surg Pathol. 2012;36(5):654.

6. Escudier B, Porta C, Schmidinger M, et al. Renal cell carcinoma: ESMO Clinical Practice Guidelines for diagnosis, treatment and follow-up. Ann Oncol Off J Eur Soc Med Oncol. 2016;27(suppl 5). 
7. Motzer RJ, Tannir NM, McDermott DF, et al. Nivolumab plus Ipilimumab versus Sunitinib in Advanced Renal-Cell Carcinoma. N Engl J Med. 2018; 378(14):1277-90

8. Choueiri TK, Lim ZD, Hirsch MS, et al. Vascular endothelial growth factortargeted therapy for the treatment of adult metastatic Xp11.2 translocation renal cell carcinoma. Cancer. 2010;116(22):5219-25.

9. Malouf GG, Camparo P, Oudard S, et al. Targeted agents in metastatic Xp11 translocation/TFE3 gene fusion renal cell carcinoma (RCC): a report from the Juvenile RCC Network. Ann Oncol. 2010;21(9).

10. Malouf GG, Monzon FA, Couturier J, et al. Genomic heterogeneity of translocation renal cell carcinoma. Clin Cancer Res. 2013;19(17):3825

11. Choueiri TK, Fay AP, Gray KP, et al. PD-L1 expression in nonclear-cell renal cell carcinoma. Ann Oncol. 2014;25(11):2178-84.

12. Motzer RJ, Escudier B, McDermott DF, et al. Nivolumab versus everolimus in advanced renal-cell carcinoma. N Engl J Med. 2015:373(19):1803-13.

13. Miao D, Margolis CA, Gao W, et al. Genomic correlates of response to immune checkpoint therapies in clear cell renal cell carcinoma. Science. 2018;359(6377):801-6.

14. Cheng DT, Mitchell TN, Zehir A, et al. Memorial Sloan Kettering-integrated mutation profiling of actionable cancer targets (MSK-IMPACT): A hybridization capture-based next-generation sequencing clinical assay for solid tumor molecular oncology. J Mol Diagn JMD. 2015;17(3):251-64.

15. Malouf GG, Su X, Yao H, et al. Next-generation sequencing of translocation renal cell carcinoma reveals novel RNA splicing partners and frequent mutations of chromatin-remodeling genes. Clin Cancer Res. 2014;20(15).

16. Cancer Genome Atlas Research Network. Comprehensive molecular characterization of clear cell renal cell carcinoma. Nature. 2013;499(7456):43.

17. Tolcher AW, Sznol M, Hu-Lieskovan S, et al. Phase Ib Study of Utomilumab (PF-05082566), a 4-1BB/CD137 Agonist, in Combination with Pembrolizumab (MK-3475) in Patients with Advanced Solid Tumors. Clin Cancer Res. 2017;23(18).

18. Boisseau W, Touat M, Berzero G, et al. Safety of treatment with nivolumab after ipilimumab-related meningoradiculitis and bilateral optic neuropathy Eur J Cancer Oxf Engl 1990. 2017;83:28-31.

19. Koshkin VS, Barata PC, Zhang T, et al. Clinical activity of nivolumab in patients with non-clear cell renal cell carcinoma. J Immunother Cancer. 2018;6(1):9.

20. Moreira RB, McKay RR, Xie W, et al. Clinical activity of PD1/PDL1 inhibitors in metastatic non-clear cell renal cell carcinoma (nccRCC). J Clin Oncol. 2017; 35(6_suppl).

21. Choueiri TK, Fishman MN, Escudier B, et al. Immunomodulatory activity of nivolumab in metastatic renal cell carcinoma. Clin Cancer Res. 2016;22(22).

22. Roh W, Chen P-L, Reuben A, et al. Integrated molecular analysis of tumor biopsies on sequential CTLA-4 and PD-1 blockade reveals markers of response and resistance. Sci Transl Med. 2017;9(379):3560

23. Van Allen EM, Miao D, Schilling B, et al. Genomic correlates of response to CTLA-4 blockade in metastatic melanoma. Science. 2015;350(6257).

24. Lieberman TD, Michel J-B, Aingaran M, et al. Parallel bacterial evolution within multiple patients identifies candidate pathogenicity genes. Nat Genet. 2011:43(12):1275.

25. Wolfert MA, Boons G-J. Adaptive immune activation: glycosylation does matter. Nat Chem Biol. 2013;9(12):776.

26. Sansregret L, Patterson JO, Dewhurst S, et al. APC/C Dysfunction limits excessive cancer chromosomal instability. Cancer Discov. 2017;7(2):218-33.

27. George S, Miao D, Demetri GD, et al. Loss of PTEN is associated with resistance to anti-PD-1 checkpoint blockade therapy in metastatic uterine Leiomyosarcoma. Immunity. 2017;46(2):197-204.

28. Jelinic P, Ricca J, Van Oudenhove $E$, et al. Immune-active microenvironment in small Cell carcinoma of the ovary, hypercalcemic type: rationale for immune checkpoint blockade. J Natl Cancer Inst. 2018;1 10(7):787-90.

\section{Ready to submit your research? Choose BMC and benefit from:}

- fast, convenient online submission

- thorough peer review by experienced researchers in your field

- rapid publication on acceptance

- support for research data, including large and complex data types

- gold Open Access which fosters wider collaboration and increased citations

- maximum visibility for your research: over $100 \mathrm{M}$ website views per year

At BMC, research is always in progress.

Learn more biomedcentral.com/submissions 